Physica B 165\&166 (1990) 81-82

North-Holland

\title{
COMPARISON OF SHUNTED DC-SQUIDS WITH LARGE $\beta$
}

\author{
E.P. HOUWMAN, D.VELDHUIS, J.FLOKSTRA, and H.ROGALLA
}

University of Twente, Faculty of Applied Physics, Low Temperature Group, P. O. Box 217, 7500 AE Enschede, The Netherlands.

The performance of DC-SQUIDs with inductively and resistively shunted inductances is studied theoretically and experimentally and compared to the performance of a standard (unshunted) SQUID. By shunting the inductance the voltage modulation depth remains unaffected for large $\beta$. The consequences for the flux-voltage transfer and the noise performance are discussed.

\section{INTRODUCTION}

Since the description of the standard DCSQUID by Tesche and Clarke several modifications on the SQUID design were proposed to overcome the fairly strict constraint for the screening parameter $\beta=2 \mathrm{LI}_{0} / \Phi_{0} \approx 1$. $\mathrm{L}$ is the SQUID inductance, $I_{0}$ the critical current of a junction and $\Phi_{0}$ the flux quantum. Thermal noise restricts $I_{0}$ to values larger than about $4 \mu \mathrm{A}$, for practical devices operated at LHe temperature. This limits $\mathrm{L}$ to about $250 \mathrm{pH}$ at the most.

We are developing a fabrication process for DC-SQUIDs for the application to neuromagnetometry (1). In that case the SQUID is coupled to an inductance of about $1 \mu \mathrm{H}$. In the KetchenJaycox washer design this can be achleved by a planar, tightly coupled coil with many $\left(n_{1}=30-60\right)$ turns. Apart from fabrication problems, the capacitive coupling between SQUID and input circuit may cause resonances in the system, that can give rise to excess noise.

The SQUID inductance can be increased, thus $n_{i}$ decreased, by the introduction of a shunt across $L$, on the expense of some loss of sensitivity, that may be acceptable for many neuromagnetic experiments. On the other hand excess noise due to resonances may be avoided.

We are studying the properties of the standard, the resistively and the inductively shunted SQUID experimentally.

\section{THEORETICAL CHARACTERISATION}

The voltage modulation depth of a standard SQUID in the optimum bias point depends on $\beta$ as $\Delta V_{m} \approx I_{0} R /(1+\beta)(2)$, where $R$ is the shunt resistance of the junction. A simple estimate of the nolse leads to an energy sensitivity $\varepsilon /\left(2 k_{B} T \Phi_{0} / I_{0} R\right) \approx(\beta+1)^{2} / \pi^{2} \beta+\beta / 4$. This expression underestimates the noise, but describes the functional behaviour fairly well.

For the resistively shunted SQUID it was shown that $\Delta V_{\mathrm{m}} \approx \mathrm{I}_{0} \mathrm{R} / 2$ is nearly independent of $\beta$, if $\gamma=R / R_{s h}$ is about 1 or larger, where $R_{s h}$ is the shunt resistance over $L$ (3). The energy resolution is analytically obtained as $\varepsilon /\left(2 k_{B} T \Phi_{O} / I_{0} R\right) \approx 4 / \pi^{2} \beta+\beta(1+2 \gamma) / 4$. For low $\beta$ the estimate is too low, but it becomes rather good for larger $\beta$ and $\gamma>0.5$. For large $\beta$ opt $1-$ mum performance is obtained for $\gamma \approx 1$.

For the inductively shunted SQUID the flux quantisation condition can be written as (4) $\delta_{1}-\delta_{2}=\pi\left[\beta_{\mathrm{sh}}-\beta_{\mathrm{sh}}^{2} /\left(\beta_{\mathrm{p}}+\beta_{\mathrm{sh}}\right)\right] \mathrm{j}+2 \pi\left[\Phi_{\mathrm{p}} \beta_{\mathrm{sh}} /\left(\beta_{\mathrm{p}}+\beta_{\mathrm{sh}}\right)\right]$ where $\delta_{1}$ is the phase difference over junction $i$, $j$ the circulating current through the junctions and $\Phi_{\mathrm{p}}$ the flux in the large plck-up loop, formed by the loop inductance $\mathrm{L}_{p}$ and the shunt inductance $\mathrm{L}_{\mathrm{sh}}$. It is assumed that no flux is applied to the small 10op, made up of $\mathrm{L}_{s h}$ and the junctions. For $\beta_{\mathrm{p}} \gg \boldsymbol{\beta}_{\mathrm{sh}}$ and $\boldsymbol{\beta}_{\mathrm{sh}} \gg 1$ this condition is that of a standard SQUID, to which an effective flux $\Phi_{\text {eff }} \approx \Phi_{p} /\left(\beta_{p}+1\right)$ is applied, but with effective screening parameter $\beta_{\mathrm{sh}} \approx 1$. Thus $\Delta V_{m} \approx I_{0} R / 2$, but the transfer becomes $\partial V / \partial \Phi_{p} \approx\left(\partial V / \partial \Phi_{\text {off }}\right) /\left(\beta_{p}+1\right) \approx\left(\pi I_{0} R / \Phi_{0}\right) /\left(\beta_{p}+1\right)$.

The energy resolution is obtained, neglecting the mixing due to the junctions and assuming that the noise current is shunted by $L_{s h}$, as $\varepsilon /\left(2 \mathrm{k}_{\mathrm{B}} \mathrm{T} \Phi_{\mathrm{O}} / \mathrm{I}_{\mathrm{o}} \mathrm{R}\right) \approx 0.35\left(\beta_{\mathrm{p}}+1\right)^{2} / \beta_{\mathrm{p}}$

Figure 1 shows the voltage modulation depth as function of $\beta$, respectively $\beta_{p}$, for the standard and the shunted SQUIDs, calculated from an analytical model based on the work of Enpuku et al. Theoretically the effect of the shunting is very pronounced in the used approximations.

Figure 2 shows the energy resolution according to the above approximate formulas. In all three cases $\varepsilon$ shows a clear minimum for $\beta \approx 1$ and a linear increase with $\beta$ for larger $\beta$. The price for larger $\beta$ is some increase of $\varepsilon$, that is comparable for the three designs.

\section{FABRICATION AND EXPERIMENTAL RESULTS}

10 SQUIDs of the three designs, are fabricated in a single run. The SQUID washer is formed by a $\mathrm{Nb} / \mathrm{Al}, \mathrm{AlO} \mathrm{O}_{\mathrm{x}} / \mathrm{Nb}$ tri-layer process, using DC magnetron sputtering and thermal oxidation. The junction areas $\left(5 \times 5 \mu^{2}\right)$ are defined by a standard SNAP process, by which also an insulation layer is formed on the washer. The employed 


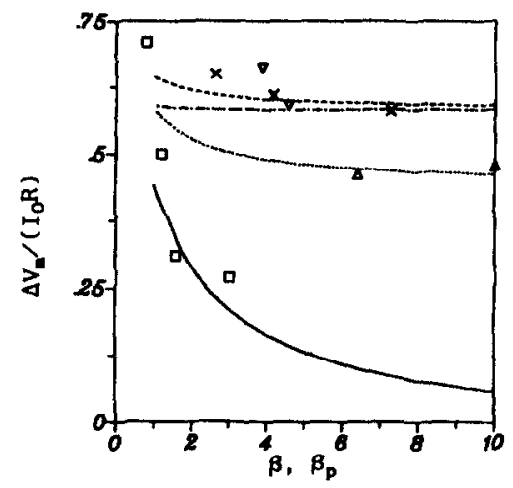

Fig. 1 : Voltage modulation depth as function of $\beta$ for noiseless SQUIDs with $\beta_{\mathrm{c}}=0$, at $I_{\mathrm{B}}=2.1 I_{0}$. Lines : theory, symbols : experimental data. - $\square$ standard SQUID; -.-.-, $x$ resist.shunted SQUID $(\gamma=1) ; \quad \ldots \ldots$ induct.shunted SQUID $\left(\beta_{\mathrm{sh}}=1\right), \cdots, \nabla\left(\beta_{\mathrm{sh}}=0,5\right)$.

junctions are fabricated with a double oxide barrier. The junctions are of high quality with $V_{\mathrm{m}}$ up to $80 \mathrm{mV}$ at $4.2 \mathrm{~K}$ and $600-1100 \mathrm{mV}$ at 1.6 K. The fabrication and characterisation of the junctions is described elsewhere (5). A second insulation and planarisation layer of $\mathrm{RF}$ sputtered $\mathrm{SiO}_{2}$ is deposited. The final $\mathrm{Nb}$ layer is patterned to form the contacts to the counter electrode of the junctions and the modulation and signal coils.

The resistively shunted SQUIDs have inductances 240, 470, 1200 and $2400 \mathrm{pH}$. We chose $\gamma \approx 1$ and $R$, such that $\beta_{c}=2 \pi \mathrm{I}_{0} R^{2} C / \Phi_{0} \leq 0.3$, throughout.

The two standard SQUIDs have inductances 110 and $240 \mathrm{pH}$. The junctions are placed at the inside of the washer to avoid the parasitic slit inductance.

The inductively shunted SQUIDs consist of a small washer with a hole inductance of $110 \mathrm{pH}$, on which the modulation coil is placed. This washer is attached to a large washer, with an inductance of 940 or $2400 \mathrm{pH}$, on which the input coil is placed.

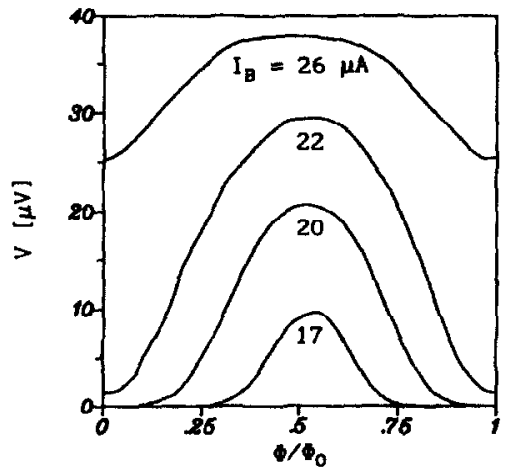

Fig. 3 : Experimental flux-voltage transfer of inductively shunted SQUID.

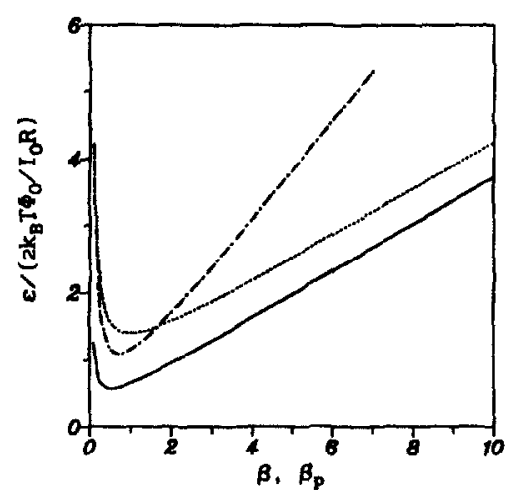

Fig. 2 : Energy sensitivity as function of $\beta$. - standard SQUID; -...- resist. shunted SQUID $(\gamma=1) ; \ldots . . . . . . .$. induct. shunted SQUID $\left(\beta_{\mathrm{sh}}=1\right)$.

In figure 1 some first results on the experimental $\Delta V_{\mathrm{m}}$ (at $\mathrm{I}_{B} \approx 2.1 \mathrm{I}_{0}$ ), obtained for the three SQUID designs, are shown. The strong dependence of $\Delta \mathrm{V}_{\mathrm{m}}$ on $\beta$ for the standard SQUID is clearly seen, in contrast with the relative independence of $\Delta V_{\mathrm{m}}$ on $\beta$ for the shunted SQUIDs.

Figure 3 shows the flux-voltage transfer of a typical inductively shunted SQUID, with $\beta_{\mathrm{p}} \approx 10, \beta_{\mathrm{sh}} \approx 1.2, I_{0} \approx 11 \mu \mathrm{A}$ and $\mathrm{R} \approx 4.3 \Omega$. The large voltage swing and the high symmetry of this curve, make this SQUID very sultable for application in flux-locked loop systems.

The sensitivity is still to be determined and will be reported elsewhere.

\section{CONCLUSIONS}

The dependence of $\Delta V_{m}$ and $\varepsilon$ of the standard, resistively and inductively shunted SQUIDs on $\beta$ is calculated. For the shunted SQUIDs $\Delta V_{m}$ is nearly independent of $\beta$, in contrast with the strong dependence for the standard SQUID. The dependence of $\varepsilon$ on $\beta$ is comparable for the three designs.

A fabrication process for high-quality $\mathrm{Nb} /-$ $\mathrm{Al}, \mathrm{AlO}_{\mathrm{x}} / \mathrm{Nb}$ tunnel junctions $\left(\mathrm{V}_{\mathrm{m}}\right.$ up to $80 \mathrm{mV}$ at $4.2 \mathrm{~K}$ and over $600 \mathrm{mV}$ at $1.6 \mathrm{~K}$ ) was developed and applied to standard and shunted DC-SQUIDs.

The $\Delta V_{m}$ of the SQUIDs was determined as function of $\beta$ and is found to be in accordance with the theoretical expectations.

\section{REFERENCES}

(1) H.J.M.ter Brake et al., Design and Construction of a 19-Channel DC-SQUID Neuromagnetometer, this Volume.

(2) R.L.Peterson and C.A.Hamilton, J.Appl. Phys.50, (1979) 8135.

(3) K.Enpuku et al., J.Appl.Phys.57, (1985) 1691.

(4) H.Koch, ICEC 10, (1984) 834 .

(5) E.P.Houwman et al., accepted for publ. in J.Appl. Phys. (1990).

D.J.Adelerhof et al., Conductance Studies on Different Types of $\mathrm{Nb} / \mathrm{AI}, \mathrm{AIO}(/ \mathrm{AI}) / \mathrm{Nb}$ Josephson Tunnel Junctions, this Volume. 